\title{
THE BLACK SILICON METHOD VI: HIGH ASPECT RATIO TRENCH ETCHING FOR MEMS APPLICATIONS
}

\author{
Henri Jansen, Meint de Boer, and Miko Elwenspoek
}

MESA Research Institute, University of Twente, P.O. Box 217, 7500AE Enschede, The Netherlands

Phone: X-31-53-4892640; Secr.: X-31-53-4892751; Fax:X-31 53-4309547; E-mail: H.V.Jansen@eltn.utwente.nl

\begin{abstract}
Etching high aspect ratio trenches (HART's) in silicon is becoming increasingly important for MEMS applications. Currently, the most important technique is dry reactive ion etching (RIE). This paper presents solutions for the most notorious problems during etching HART's: tilting and the aspect ratio dependent etching effects such as bowing, RIE lag, bottling, and micrograss or black silicon. To handle these problems submicron HART's are etched and the black silicon method is used to direct the pressure, power, ion energy, or flows of a fluorine-based RIE into the preferred settings. The influence of ion energy and -trajectory is found to be most critical. The behaviour of the HART process is explained with the help of a set of variables and used to optimise the final profile. After this optimisation the RIE setting found is used for etching supermicron HART's which are characteristic for MEMS applications.
\end{abstract}

\section{INTRODUCTION}

In the first years after the introduction of dry plasma etching in microelectronics, the technique was mainly used for the ashing of photoresist because of its cleanliness and high selectivity [1]. In the beginning, the isotropic nature of these so-called plasma etchers (PE) was not a problem but the ever decreasing dimensions in the integrated circuits forced the research institutes to develop dry plasma systems for anisotropic etching and the ion beam etching (IBE) was born [2]. However, the etch selectivity between mask-substrate was rather poor and special IBE reactors e.g. chemical assisted ion beam etching (CAIBE) were designed [3].

A major step into the direction of large scale integration was taken after the reactive ion etching (RIE) came available [4]. In RIE it is possible to get a very high selectivity with a perfect anisotropy [5]. Nowadays, to increase the electronic circuit density, the use of the surface area of the silicon wafers only is not sufficient and the research is focused to use the third dimension: depth. So, there is an increasing demand for processes which are able to create not only a high selectivity and anisotropy but also high aspect ratio trenches (HART's). The aspect ratio (AR) is defined as the maximum depth divided by the maximum width. Such HART's, e.g. a quarter micron in width and three micron in depth, are needed for e.g. transistor trench isolation and trench capacitors. HART's are not only important for silicon etching. Especially the etching of HART's in polymers is a prime technology [6].

In micromechanics, as in microelectronics, profile control of HART's is increasingly important. These trenches give rise to specific problems: The aspect ratio dependent etching (ARDE) effects and tilting [7-9]. In figure 1 these effects are shown: RIE lag, bowing, and trench area dependent tapering of profiles (TADTOP, fig.1a), bottling (fig.1b), micrograss (fig.1c), and tilting (fig.1d). The main subject of this study is to explain and control these effects.

The RIE process is analysed with the help of four sets of variables: i) The RIE setting, ii) the equipment parameters, iii) the plasma characteristics, and iv) the trench forming mechanisms. These figures are controlling the final HART profile. The study continuous with information concerning the equipment: a conventional RIE of the STS company and a cryogenically cooled RIE with a high density source of the Plasma Technology company. After information about the sample preparation, results and discussions are given for the HART's which are etched. Because the tapering of the trenches is changing with RIE setting, the black silicon method (BSM) is used to be able to change the setting while keeping the profile anisotropic [10].
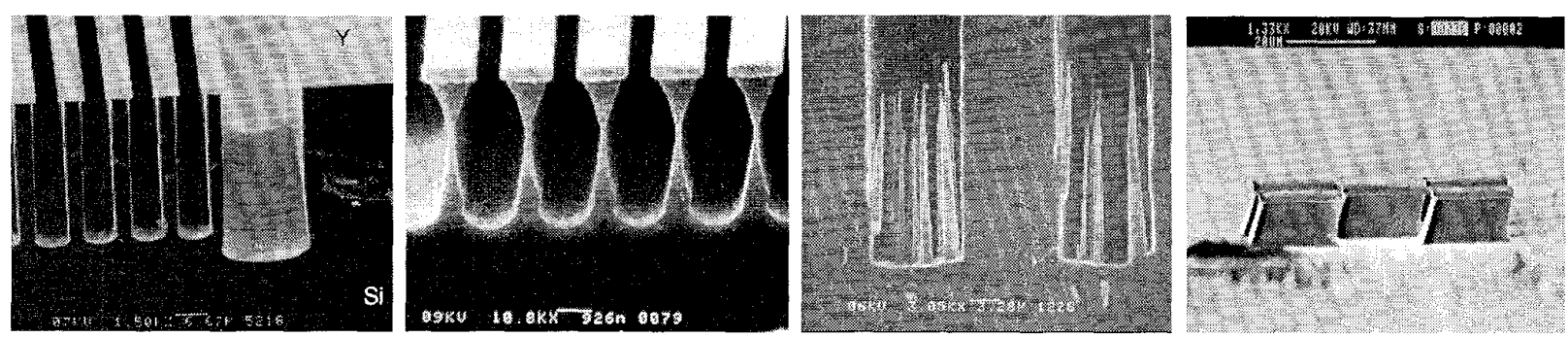

Figure 1: important ARDE effects. a) RIE lag, bowing, and TADTOP, b) bottling, c) micrograss, and d) tilting. 


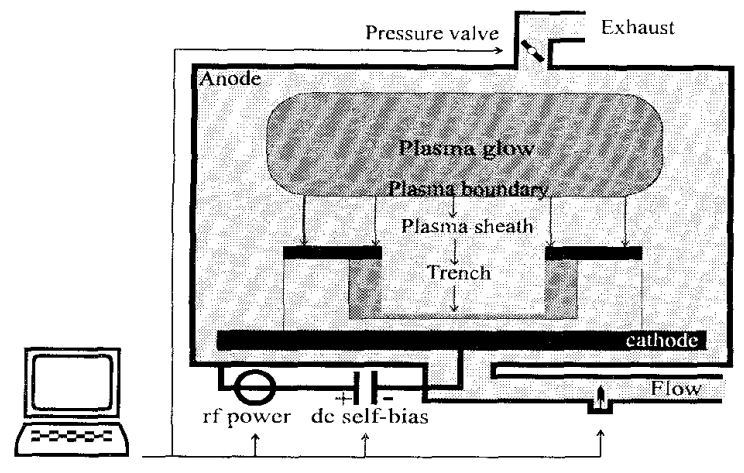

Figure 2: Basic RIE system.

\section{QUALITATIVE ANALYSIS}

There are two fluxes of particles of major concern in RIE: ions and radicals. These particles are the main source for etching. Together with the neutral density they determine how the final profile will evolve in time during etching i.e. they determine the HART effects. To get an idea of the behaviour of the particle fluxes and energies, the etch process is split into four sets of variables as found in figure 2: the i) RIE setting, ii) equipment parameters, iii) plasma characteristics (glow, boundary, and sheath), and iv) trench forming mechanisms. The HART effects are explained while using these variables. The RIE sctting is controlled by the opcrator whereas the equipment parameters are determined by the manufacturer. The plasma characteristics are depending on the RIE setting and the equipment. Together with the trench forming mechanisms these figures are responsible for the final result of the trench profile. In this section these sets of variables are qualitatively highlighted.

RIE setting: This set is directly controlled by the operator. It consists of the familiar pressure, flow, power, and temperature. Sometimes it is possible to create a dc self-bias which is not directly controlled by the rest of the parameters. For example, when the reactor geometry is changed (showerhead) or when an extra plasma source (ICP) is used, the dc self-bias will be an independent variable. Another parameter controlled by the operator is the sample to be etched. This can be a conducting material like silicon but also an insulating polymer. There are some variables depending on the other settings. The residence time $\mathrm{t}_{\mathrm{r}}[\mathrm{s}]$ is one of them and expressed in the others as $t_{r} \sim p V / Q$ with $p$ the operation pressure, $\mathrm{V}$ the reactor volume, and $\mathrm{Q}$ the total flow.

Equipment parameters: The manufacturer of an RIE system determines most boundary conditions for the RIE setting. For example, when a high flow is needed at a low operation pressure the manufacturer is forced to imbed a vacuum system able to handle such flows e.g. a turbopump with a high throughput. Other examples are the anode/cathode ratio and the rf frequency which are responsible for the magnitude of the dc self-bias created by the plasma.

Plasma characteristics: These variables are a function of the RIE setting and the equipment parameters. In this section a closer look at the plasma is given. Most plasma reactors consist of two electrodes connected to an rf voltage source which enclose a low pressure gas as a dielectric. The $\mathrm{rf}$ electrical field will force electrons to the positive electrode. In their way they will collide with the feed gas generating the gas phase etching environment which consists of neutrals $(\mathrm{N})$, radicals $(R)$, electrons $(E)$, ions $(I)$, photons $(P)$, and phonons (T). The photons are responsible for the characteristic glow of the plasma. Since the electron mobility is much greater than the ion mobility the electrons are able to track the rf electrical field. So, after ignition of the plasma the electrodes acquire a negative charge whereas the plasma becomes positively charged. Of course, only electrons in the direct neighbourhood of the electrodes will reach them during the $\mathrm{rf}$ cycle. Therefore, a thin region depleted from electrons will be developed close to the electrodes: The plasma sheath. Because there are no electrons in this region to generate photons, the region is dark and the plasma sheath is also known as the dark space. The rest of the plasma is called the glow region. Both regions are separated clearly by the so-called boundary layer. Shortly, a plasma can be divided into three components: i) The glow region full of electrons, ii) the sheath region depleted from electrons, and iii) the boundary layer separating both regions. Due to the charging of the glow region a dc electrical field will exist in the sheath region forcing positive ions to the electrode. This phenomena is typical for RIE: A continuous flow of directed ions together with an isotropic flux of radicals. In most cases, the area of the two electrodes facing the plasma are not the same. Therefore, a different negative charging of the electrodes is a result and the $\mathrm{dc}$ voltage between the glow and one of the electrodes will differ from the other one. This difference in de voltage between the two electrodes is called the de self-bias which is a measure of extreme importance in RIE. The electrode having the biggest area facing the plasma is always at a higher dc potential than the smaller one and is called the anode. The smaller electrode is known as the cathode.

The glow region: This region is characterised by a set of glow variables: The particle densities $\left(\rho_{\mathrm{N}}, \rho_{\mathrm{R}}, \rho_{\mathrm{I}}, \rho_{\mathrm{E}}, \rho_{\mathrm{P}}\right.$, and $\rho_{\mathrm{T}}$ ) and the energy of these particles (expressed in $\mathrm{eV}$ or $\mathrm{K}$ where $1 \mathrm{eV}=8000 \mathrm{~K}$ ). Other examples are the power- and energy density, and the floating potential: i.e. the potential of a floating sample in the glow region with respect to the anode potential. The electrons are the only particles able to gain energy from the $\mathrm{rf}$ power source. Therefore, an important variable is the electron energy distribution function (EEDF, fig. 3 a) which is a measure of the energy of the electrons. Generally, the electrons will collect energy between 3 and $30 \mathrm{eV}$ before colliding with another particle which is strongly depending on the operation pressure of the RIE. A typical particle generated by electron impact is the photon. The energy of the photon (expressed in $\mathrm{eV}$ or $\mathrm{nm}$ where $1 \mathrm{eV}=1200 \mathrm{~nm})$ is depending on the electronic configuration of the bombarded particle and therefore the photon energy distribution function (PEDF, fig.3b) is related to the feed gas. The total spectrum of photons are giving the plasma a typical colour. For example, the colour of a nitrogen plasma is pinkish whereas an oxygen plasma generally is greyish-white.

The boundary layer: The energetic particles from the plasma glow should be transported to the sample surface. This is accomplished by a flux of particles through the plasma boundary layer. The radicals are having only thermal energy and are leaving the surface in all directions i.e. isotropically. However, because the glow is a conductor, the electrical field is pointing exactly from the boundary surface and the ions will leave the boundary layer under an angle of 90 degrees. 

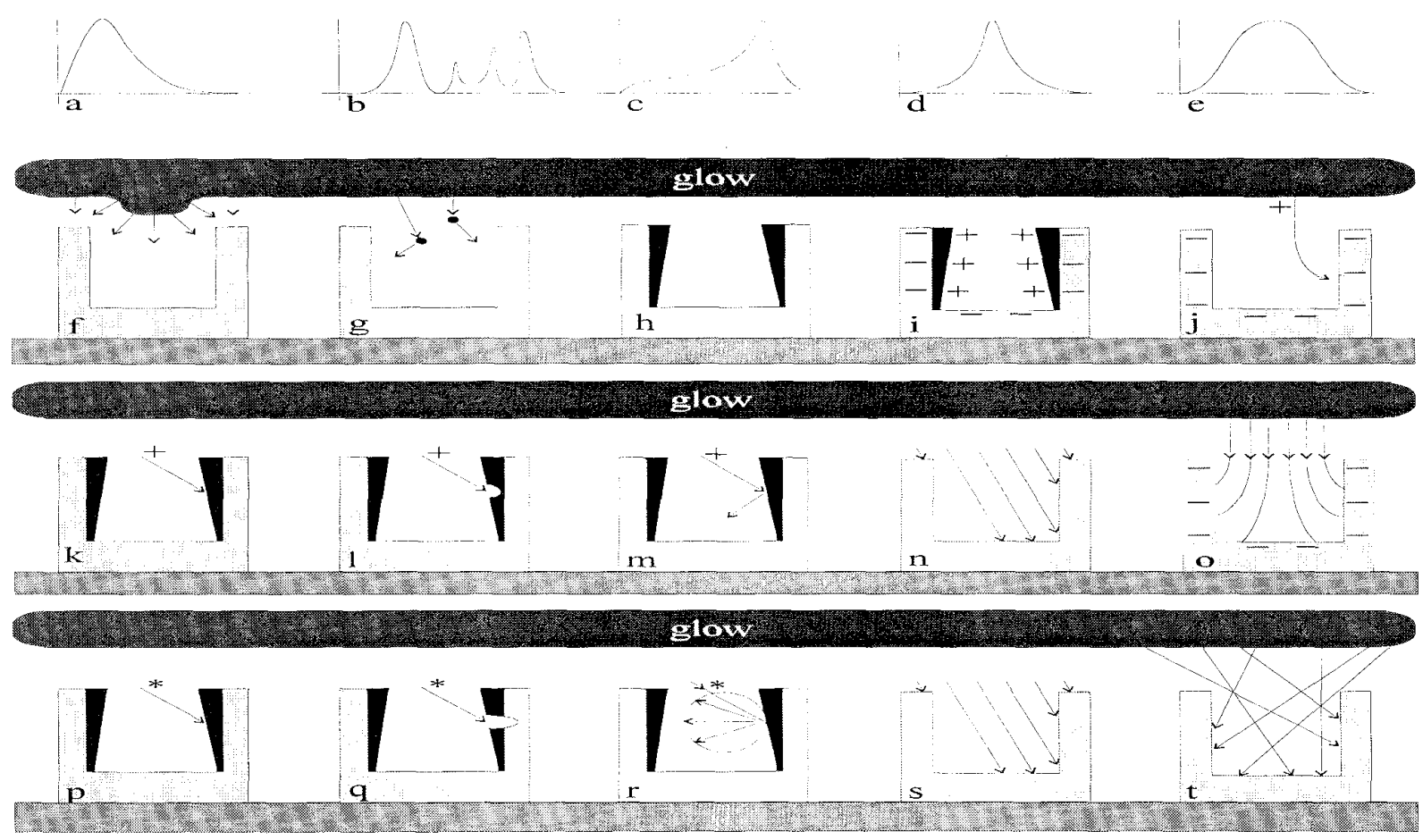

Figure 3: Distribution function and trench forming mechanisms. a) EEDF, b) PEDF, c) IEDF, d) IADF, e) RADF,

f) boundary distortion, $g$ ) particle collision, h) sidewall passivation, i) sidewall charging, $j$ ) ion deflection, $\mathrm{k}$ ) ion capturing, 1 ) ion etching, $\mathrm{m}$ ) ion reflection, $\mathrm{n}$ ) ion shadowing, $\mathrm{o}$ ) ion depletion,

p) radical capturing, q) radical etching, r) radical reflection, s) radicai shadowing, and t) radical depletion.

The sheath region: Both radicals and ions will collide with gas particles during passing the sheath. Especially, the effect of the pressure on the ions is crucial [17]. Because of the collisions of ions with other particles, ion dispersion will occur i.e. their direction will not exactly correspond with the normal of the boundary layer anymore. This effect is expressed with the help of the ion angular distribution function (IADF, fig. $3 \mathrm{~d}$ ). At the same time, the energy of the ions is exchanged with the particles and this effect is found in the ion energy distribution function (IEDF, fig.3c). The rf frequency is responsible for a varying plasma potential. So, the energy of an ion is depending on the time that the ion enters and leaves the boundary layer. This effect should be incorporated in the IEDF also. Saying it differently, the IADF is a measure for the degree of collimation or dispersion of the flux of ions. A sharp IADF means that most ions travel in the same direction. The IEDF is a measure for the energy content of the ions. A sharp IEDF means that most ions arrive with the same energy at the sample surface. Generally, the IADF and IEDF are strongly correlated i.e. they can't be varied independently.

In most cases, the collision of radicals with other particles is not important because, generally, this flux is already isotropic. However, during extensively etching silicon there will be a continuous net flow of radicals from the non-etching surrounding to the etching areas. Of course, this flow will be directional and the radical angular distribution function (RADF) becomes a meaningful expression (fig.3e). When there is a directional radical flux the RADF is broadened due to radical collisions.
Trench forming mechanisms: The flux of particles from the plasma glow region are used to etch a sample. For example, this paper discusses the $\mathrm{SF}_{6} / \mathrm{O}_{2}$ - $\mathrm{Si}$ system, which is an ion-inhibitor process, to explain the mechanisms during etching HART's. In this system the oxygen is passivating the silicon surface with siliconoxide and the $\mathrm{SF}_{5}{ }^{+}$ions are etching the passivator making it possible for the $\mathrm{F}^{*}$ radicals to etch the silicon. During etching HART's specific mechanisms which control the profile are important such as ion deflection, radical depletion, and wall passivation. These mechanisms are a function of the other variables and the AR of the HART's.

Boundary distortion (fig. $3 \mathrm{f}$ ) is found when a sample is placed on top of the cathode. This sample will distort the boundary layer which will adapt its form conform the sample geometry. Therefore, ions will leave the boundary with an off-normal angle with respect to the cathode surface. This effect is pronounced when the sample/trench geometry is greater than the thickness of the sheath region.

Particle collision (fig. $3 \mathrm{~g}$ ) is caused by gas molecules in the sheath region. The mean free path of particles is ca. $50 \mu$ $\mathrm{m}^{*}$ Torr i.e. $5 \mathrm{~cm}$ at $1 \mathrm{~m}$ Torr or $0.5 \mathrm{~mm}$ at $100 \mathrm{~m}$ Torr. These collisions are the main reason that the RADF, IADF, and IEDF are necessary functions to know in the RIE process. Particle collisions with gas molecules in the trench could be found when the pressure is high due to reaction products. However, the reaction product is $\mathrm{SiF}_{4}$ thus completely depending on the radical flux from the glow region. This flux is always much lower than the neutral flux. Therefore, the pressure in the trench always equals the operation pressure and we only have to consider the RADF, IADF, and IEDF. 
Sidewall passivation (fig. $3 \mathrm{~h}$ ) is necessary in ion inhibitor RIE processes to achieve anisotropy. For anisotropic etching, it is necessary that the horizontal etching (undercut) is as small as possible whereas the vertical etching i.e. the etch rate should be large. For the $\mathrm{SF}_{6} / \mathrm{O}_{2}$-Si system, the horizontal etching is depending on the thickness of the passivating layer and the F-atom density trying to etch the silicon by penetrating this layer. The thickness of this layer is a function of e.g. the O-atom density, the ion impact, and the local temperature. The F-atom density is a function of e.g. the $\mathrm{SF}_{6}$ flow, power, and (micro)loading.

Sidewall charging (fig.3i) will occur when the sidewall inhibitor is an insulator. Ions colliding with the sidewall will leave their charge which is difficult to compensate with electrons from the silicon behind the inhibitor. This charge will create an electrostatic field which repels a next ion.

Ion deflection (fig. $3 \mathrm{j}$ ) is caused by the diffraction of ions while entering a trench but mainly by the negative potential of conducting trench walls with respect to the plasma glow resulting in an electrostatic deflection of these ions to the walls $[11,12]$. It is concluded in this paper that ion deflection is the driving force behind most ARDE effects.

Ion capturing (fig. $3 \mathrm{k}$ ) is found when the created dangling bond isn't used for a fluor atom and an oxygen radical is connected to the free bond. In this case the product isn't volatile and there is no net etching. The battle between the fluor- and oxygen radicals is depending on how strong the passivation can be. For example, the surface mobility of Fatoms is decreasing with temperature although its lifetime at the surface increases. Therefore, when an ion has created a dangling bond, the F-atom might be too late to fill the vacant place. Instead, oxygen gas will take in the place.

Ion etching (fig.31) happens when the ion isn't reflecting. In most cases, the highly energetic ion will remove the inhibitor and create dangling bonds for the Si atoms. These bonds can be connected to fluor radicals, which will remove the $\mathrm{Si}$ because $\mathrm{SiF}_{4}$ is volatile.

Ion reflection (fig. $3 \mathrm{~m}$ ) will occur when ions collide with a surface under a glancing angle. In principle, the chance reflection takes place will follow a cosine rule as found in the literature about ion beam etching. When the incoming ion is kinetically highly energetic, the reflection will be specular. Clearly, ion reflection is broadening the IADF in the trench.

Ion shadowing (fig. $3 \mathrm{n}$ ) is caused by the top-side of the trench. When ions arrive under an angle the top-side of the trench will block ions from etching a part of the trench sidewall. In other words, the amount of ions arriving at the sidewall is depending on its position: In the higher regions the ions from approximately half the IADF will be captured by the wall. In contradiction, in the lower regions the IADF will be sharpened more and more because ions arriving at a high angle with respect to the sample surface normal are blocked by the trench top-side. Thus, the ion shadowing is responsible for the sharpening of the IADF in the trench.

Ion depletion (fig.3o) due to ion etching or capturing is an important parameter to achieve HART's. Ions collected by the sidewall of a HART can't be used for the vertical etching. The relative amount of ions arriving at the trench bottom with respect to the total incoming flux is depending on the AR only. Therefore, the etch rate is decreasing with the AR of a
HART and thought to be responsible for most ARDE effects found in this study, but in particular for RIE lag.

Radical capturing (fig. $3 p$ ) is found when the radical hits a wall. This radical will spend a certain time at the surface before it is connected to a dangling bond (etching) or is leaving again (reflection). The time a radical gets to find such a place before it will desorb is $\tau=\tau_{0} \exp \left(\mathrm{E}_{\mathrm{ads}} / \mathrm{kT}\right)$ with $\mathrm{E}_{\mathrm{ads}}$ the energy for adsorption and $\tau_{0} \sim 10^{-13} \mathrm{~s}$ the characteristic atomic thermal vibration time of a solid. In other words, the time that a non-etching radical will spend on a surface is decreasing when the temperature is increasing. Meanwhile, it is possible for the radical to move along the surface. This surface mobility is depending on the surface temperature. The higher the temperature the higher the surface mobility.

Radical etching (fig. $3 q$ ) is possible due to penetration of the inhibitor or when the inhibitor is removed by way of ion etching leaving the silicon unprotected. When four fluor atoms are connected to a silicon atom, this molecule is able to desorb from the surface because it is volatile.

Radical reflection (fig. $3 \mathrm{r}$ ) is found when the radical wasn't able to find a dangling silicon bond in time. This radical will desorb from the surface in a random direction not depending on its direction before collision. In most cases during RIE trench etching, the mean free path of particles is higher than the dimensions of the trench. Therefore, gas collisions in the trench are unlikely and we have to consider collisions with the sidewall only i.e. molecular flow. The flow resistance of a trench is caused by the random reflection of a low energetic particle, such as radicals, against the sidewall. Therefore there is only a small chance for a particle to enter the trench. This chance is known as the Clausing factor $C$ and is depending on the $A R: C(A R)=(1+0.4 A R)^{-1}$ for a tube. Clearly, this effect may cause RIE lag. For the highly energetic ions the reflection is specular and therefore the Clausing factor is 1 i.e. the ions which enter a trench will not be back scattered.

Radical shadowing (fig.3s) is identical with ion shadowing and is important because the radical flux is almost isotropic although random reflections balance this effect.

Radical depletion (fig. $3 t$ ) is found as the well-known microloading effect. It causes HART's of Si in open areas to etch at a slower speed than HART's with the same dimensions located in areas where most Si surfaces are shielded from the plasma with the help of a mask. The same mechanisms causing ion depletion, except for ion deflection, is causing radical depletion. So, only radical reflection or a great surface mobility may transport radicals into the lower trench region and radical depletion might cause RIE lag problems because radicals are consumed in the higher regions of the trench.

HART effects: The given variables are controlling how the final profile will evolve. However, because the trench forming mechanisms are a function of the other parameters it is necessary and enough to consider these mechanisms only.

\section{EQUIPMENT \& EXPERIMENTAL}

In order to track and categorise important ARDE effects, two different RIE reactors where used. The first is a conventional RIE of the STS company used for the high pressures [13]. For the low pressure experiments a dedicated high density RIE is used build by the Plasma Technology company [14]. 

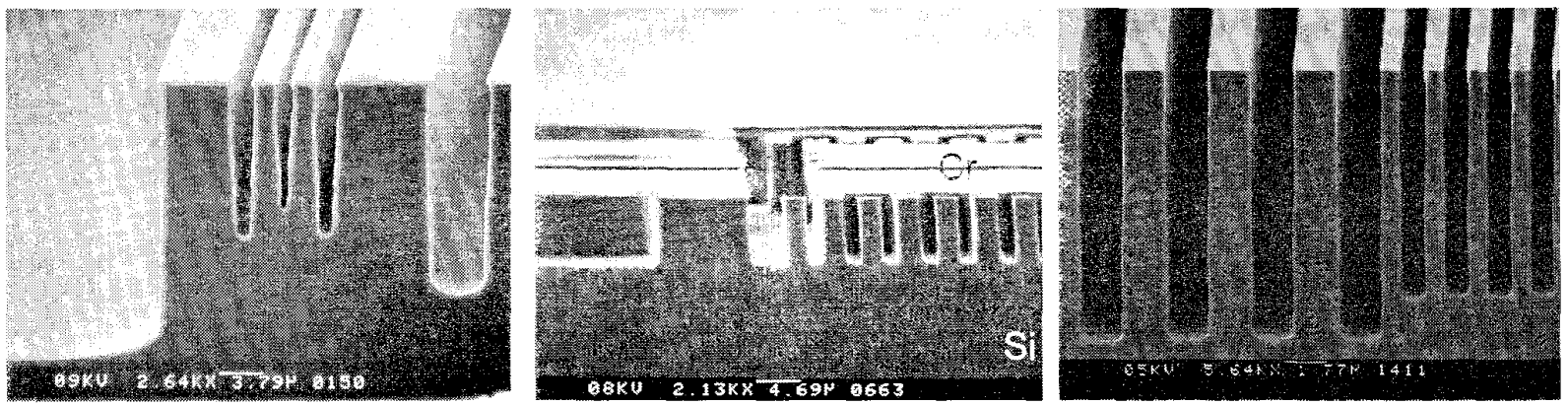

Figure 4: Identical profiles for different trench openings. Figure 5: Decreasing the ARDE effect due a stronger passivation. Figure 6: RIE lag with ICP-RIE

Plasmafab 310/340: This reactor has been used for the high pressure experiments. Information about the equipment can be found in ref.15. This RIE was modified with a showerhead placed into the reactor. The main purpose of this is to minimise the area ratio between the anode and the cathode surfaces. This change decreases the do self-bias, thus the ion energy, which is characteristic for RIE equipment. Although the showerhead will affect the other plasma parameters also (e.g. the flow resistance of the showerhead increases the operating pressure), it is assumed that these changes are minor in comparison to the change in ion energy.

Plasmalab 100: This system features a strong 1000 litres per second turbopump connected to the reactor with huge $20 \mathrm{~cm}$ pipelines to ensure a low operation pressure (10mTorr). An inductively coupled plasma (ICP) source is used to create enough plasma particles at such low pressures. An extra matching unit is used to drive the reactor in the RIE mode creating a dc self-bias. Therefore it is possible to control independently the particle flux coming from the ICP source and the ion energy due to driving the reactor in the RIE mode. To increase the passivation capability of the oxygen at the trench sidewalls and to ensure a stable wafer temperature, wafers are floating on a thin film of helium cooled down to minus $150^{\circ} \mathrm{C}$ and up to $200^{\circ} \mathrm{C}$.

Experimental: To study the profile development in HART's, sub- and super-micron patterns where deposited on top of silicon wafers. Silicon wafers, 4 inches in diameter, with submicron $250 \mathrm{~nm}$ thick aluminium patterns (down to a quarter micron in width) where supported by the Philips Research Lab in Eindhoven. The supermicron patterns where gratings with a period of $1,2,5$, and 10 micron. As a mask a $20 \mathrm{~nm}$ chromium layer served applied with the help of the liftoff technique. Both batches didn't get any special treatment to remove dirt or native oxide. Samples to be etched with an $\mathrm{SF}_{6} / \mathrm{O}_{2}$ plasma where carefully attached to the target platen.

\section{HART's}

In figure 4 a typical cross section of some trenches is shown. It can be seen that the shape is identical, not depending on the width. Note that this statement is not always true as observed in fig.la where the TADTOP effect is seen). This observation enables us to use submicron patterns instead of the largersized patterns. After optimising HART's with the help of the BSM, the parameter setting will be used to etch the supermicron gratings. This paragraph will treat tilting and the ARDE effects (i.e. RIE lag due to ions or radicals, bowing, TADTOP, bottling, and micrograss) one by one and are explained with the help of the analysis already given. Every section is ended with a conclusion how the effect is caused and solutions are given to prevent these HART effects.

Tilting: This effect is observed for extremely wide trenches and at the wafer corners. It is caused by the boundary distortion which produces off-normal ions with respect to the cathode surface area. A mechanical wafer clamping (obstacle) produces the same effect. In figure Id a typical example is shown caused by boundary distortion at the wafer edge. Similarly, a difference in radical concentration might cause tilting because this concentration is controlling the radical flux coming from a certain direction. For a Si wafer placed on top of a non-etching target platen, a directional radical flux is flowing from the direct surrounding of the Si wafer to the wafer centre. Therefore, tilting is possible even when there is no boundary distortion.

Bowing: In figure 1a a typical example of bowing is observed. In the wider trench a parabolic curvature of the etched wall (i.e. negatively tapered) is observed. This effect is explained with the help of ion deflection due to electrostatic forces and can be minimised by increasing the sidewall passivation, the wall charging, the energy of the ions before entering the trench, or preventing these forces as in ion beam etching. This can be achieved by cryogenically cooling the sample or by letting an extra passivation gas like $\mathrm{CHF}_{3}$ or $\mathrm{O}_{2}$ into the plasma. For non-conducting samples like Teflon or siliconoxide this effect should not be observed.

The effect of bowing caused by ions can be controlled with the help of the BSM. In figure 5 the bowing has been reduced by making the silicon trench more insulating-like, thus increasing the passivator in the plasma by way of extra oxygen. To ensure a certain profile, the ion flux has to be increased at the same time by way of extra $\mathrm{CHF}_{3}$ conform the $\mathrm{BSM}$. The $\mathrm{SF}_{6} / \mathrm{O}_{2} / \mathrm{CHF}_{3}$-chemistry enables us to passivate the sidewall with siliconoxide or fluorocarbon (FC). The silicon oxide is much stronger but also thinner than the $\mathrm{FC}$ layer. Therefore, ion deflection is more pronounced for $\mathrm{SF}_{6} / \mathrm{O}_{2}$ etching, although the sidewalls are smoother as in the case of $\mathrm{SF}_{6} / \mathrm{CHF}_{3}$ etching, for $\mathrm{FC}$ is easily etched with ions [6].

TADTOP: The trench area dependent tapering of profiles is found in again figure la where the wider trench is more negatively tapered than the smaller trenches. Ions which enter a small cavity will be less attracted by its nearest wall because its opposite wall is closer than in the case of a wide opening. This wall is neutralising the closest wall and therefore the smaller opening is more positively tapered. In other words, ion deflection is responsible for this effect and the solutions for this effect are identical with those for the bowing effect (see fig.5). 

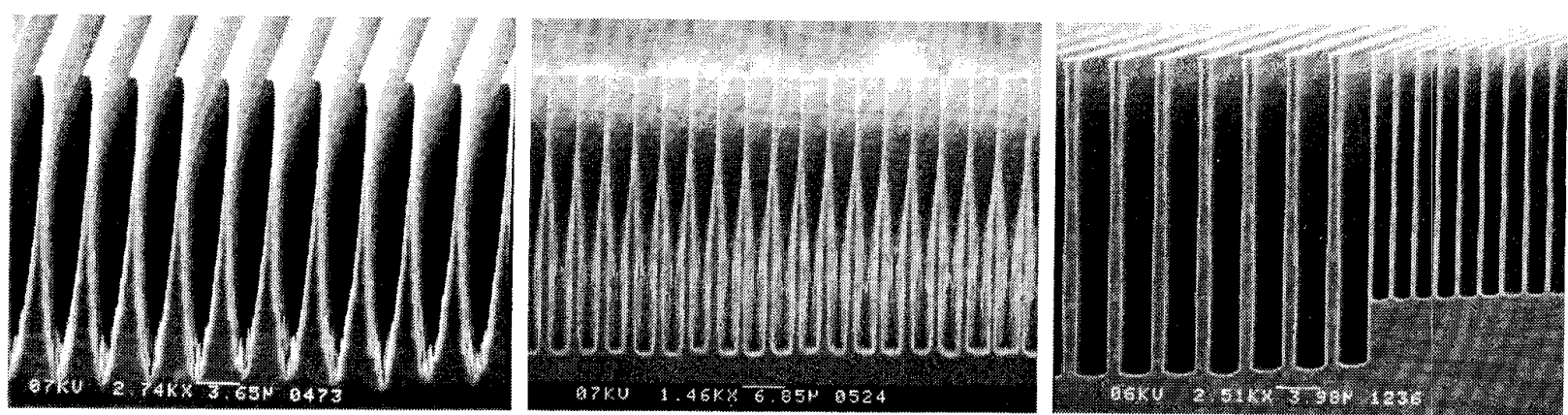

Figure 7: RIE lag due to radical depletion: a) High pressure wafer centre, b) high pressure wafer edge, and c) low pressure ICP-RIE wafer centre and edge.

RIE lag due to ions: RIE lag is associated to the effect that smaller trenches are etched faster (negative lag) or slower (positive lag) than wider trenches as found in figure 1a. Positive lag may be explained in considering the amount of ions which exist during their trajectory in the trench. During this travel the outer ions will be collected by the negative (conducting) walls due to ion deflection. This process will go on until all the ions are captured by the walls. So, at last there will be no ions left to etch the bottom and etching stops in this direction (In fact this is not completely true: During trench etching, the higher region of the trench is becoming more and more isolated due to oxidation (fig.3i). Therefore, wall charging will increase ion reflection i.e. decrease ion depletion). It is obvious that for the small trenches this ion depletion is reached sooner than for the wider because the flux/wallarea ratio is smaller after a certain etch depth (figure 1a). In fact, it can be shown easily that only the relative trench dimension (-AR) and not the absolute trench dimension are controlling the etch depth and -rate.

Ion deflection should not change the profile of insulating trenches because this effect would soon be overruled by the positive charging of the walls. Therefore, we investigated the IBE of Teflon and the RIE of SiOxNy layers [6]. During etching these layers bowing, TADTOP, and RIE lag were barely observed indicating that the conducting wall can explain these ARDE effects during etching HART's in silicon [16]. However, in literature RIE lag is without doubt observed for isolating substrate materials [7]. The reason for this discrepancy could be found in the much lower pressures at which the samples were etched. For example, Teflon is etched with the help of IBE which operates at an extremely low pressure $(<0.2 \mathrm{~m}$ Torr $)$. The siliconoxynitride was etched at approximately $30 \mathrm{~m}$ Torr which should make aspect ratios of over 10 possible. The samples were not etched that long to create an aspect ratio exceeding 10 , so radical reflection as well as ion and radical depletion are probably not yet important. Any way, the deflection of ions can be reduced by increasing the passivation. Again, in fig. 5 it is demonstrated that the RIE lag is decreased successfully.

More evidence for the depletion of ions due to ion deflection is found in figure $1 \mathrm{c}$. In this figure a 10 micron wide trench opening is observed with spikes in it. Quite clearly, the spikes are pointing to the centre of the trench. Because the direction of the spikes is an indication for the direction of the ions, it is concluded that the ions are forced to the sidewall by the electrical fields in the trench. Note that the sidewall is straight, although the ions collide under an angle.
In figure 6 a cross section of a trench etched with the help of the ICP-RIE operating at low pressure is shown. The effect of RIE lag is clearly visible. The phenomena can be explained by the deflection, capturing, and subsequent depletion of ions. Due to the strong wall passivation ions are not able to etch the inhibitor and their journey is ended at the spot where they collide with the sidewall.

RIE lag due to radical depletion or reflection: In figure 7 three cross-sections of the same channel are shown. The trenches are etched under three different circumstances. Figure $7 \mathrm{a} / \mathrm{b}$ are etched at relatively high pressure and substrate temperature $\left(100 \mathrm{~m}\right.$ Torr, $\left.10^{\circ} \mathrm{C}\right)$ and $7 \mathrm{c}$ is etched at low pressure and substrate temperature $\left(8 \mathrm{mT}\right.$ Torr, $\left.-100^{\circ} \mathrm{C}\right)$. In figure $7 \mathrm{a}$ the profile of a trench etched in the middle of the wafer is shown and figure $7 \mathrm{~b}$ shows the same channel but now located at the wafer edge. In other words, picture $7 \mathrm{a}$ is taken after etching and braking the sample whereas $7 \mathrm{~b}$ was already broken before etching. It can be observed that trenches at the edge are having an aspect ratio of approximately 20 whereas the trenches in the mid are not exceeding 10. It is thought that the lag for the trenches in the mid of the wafer is due to the depletion or reflection of radicals. At the edge the etching is not hampered because radicals will easily arrive from the surrounding. Thus, to explain the ARDE phenomena of trenches with aspect ratios beyond 10 it is not enough to consider ion depletion only. Radical depletion should vanish when the underetching caused by ion etching is made smaller. To verify this theory the same pattern was etched at low pressure and substrate temperature. Figure $7 \mathrm{c}$ shows a trench located in the mid of the wafer after braking the sample. The aspect ratio is 20 and it is found that trenches at the wafer edge are identical which means that radical reflection is not yet important. Shortly, at high AR radical consumption may cause depletion responsible for RIE lag. Radical reflection isn't found although this effect should be quite pronounced for $A R>10$. Maybe, the transport of radicals along the trench sidewall is sufficient to supply enough radicals. Notice that the effect of RIE lag is still present in figure $7 \mathrm{c}$. However, this is due to ion- and not radical depletion or reflection.

Micrograss: In figure $8 \mathrm{a} / \mathrm{b}$, two trenches etched at a relatively high pressure for a different time are shown. It is observed that the trench etched for a short time (fig.8a) is free from micrograss whereas the deeper trench (fig. $8 \mathrm{~b}$ ) is not. In both situations the open silicon area stayed smooth. The reason for this difference might be the sharpening of the IADF due to the higher AR of the deeper trench opening. So, in the beginning the ion dispersion is high enough to prevent 

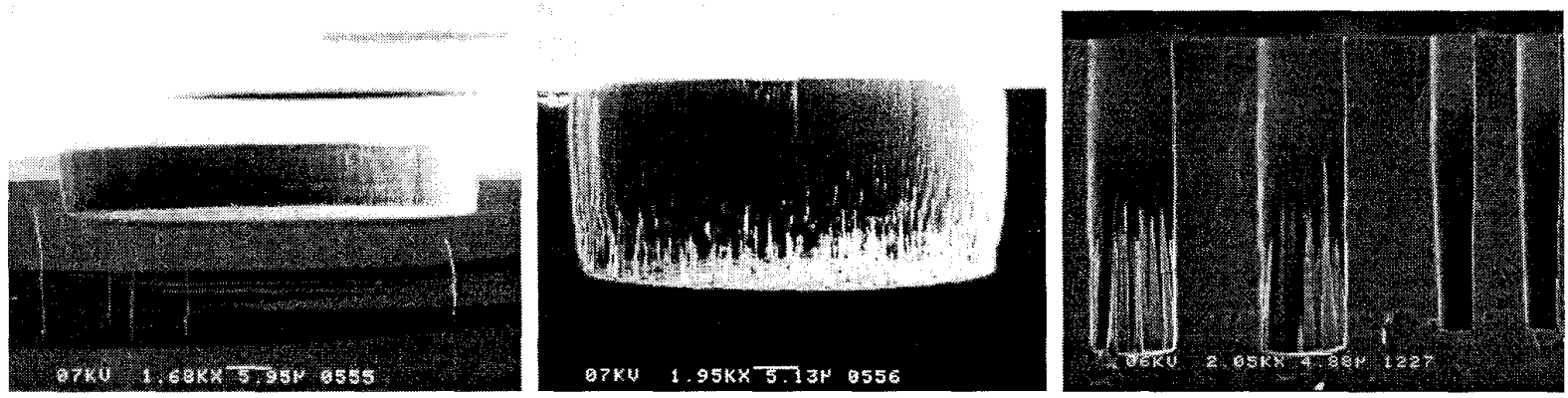

Figure 8: Micrograss as an ARDE effect: a) after $10 \mathrm{~min}, \mathrm{~b}$ ) after $20 \mathrm{~min}$, and c) prevention of-micrograss due to ion reflection.

grass but during etching the $\mathrm{AR}$ increases and the IADF sharpens enabling grass to form at the bottom. Thus grass is formed when the flux of incoming ions is highly collimated i.e. the IADF is sharp. The grass is prevented when the IADF is broadened e.g. by way of a higher pressure. The opposite effect happens when ion reflection is made possible. In figure $8 \mathrm{c}$ the smaller trench is free from grass due to the reflections of ions against the sidewall which broadens the IADF. For the wider trenches this effect is not yet enough.

Bottling: In figure $1 \mathrm{~b}$ the effect of bottling is found. Such profiles are quite common in deep trench etching and are often confused with bowing. Bowing is caused by ion deflection and is responsible for the negative slope of trenches. In contrast, the bottle shaped trenches are caused by ion shadowing and sharpening of the IADF when ions are travelling down the trench. In other words: The effect is caused by off-normal ions due to a relatively high operating pressure. In the higher regions of the profile, ions are coming in at different angles and there will be a strong undercut depending on the ion energy. However, the ions which are hitting and etching the sidewall are used up and due to the ion shadowing (i.e. blocking of incoming ions due to the opposite sidewall) the angular distribution function is sharpened. At a certain critical angle the ions are not etching the sidewall anymore but they will be captured or reflected i.e. bounce until they hit and etch the bottom of the trench. In other words, in the higher regions of the trench ions will etch the sidewall whereas in the lower regions this etching stops because these ions will fail due to ion shadowing and ion depletion. Therefore, ion shadowing and ion depletion are thought to be responsible for the bottling effect.

To prevent the bottling effect it is possible i) to sharpen the IADF by way of e.g. decreasing the pressure or ii) to decrease the IEDF by way of e.g. the de self-bias. In figure 9 an example of this last technique is shown. The parameter setting for both pictures is the same except that in figure $9 \mathrm{~b} \mathrm{a}$ showerhead is mounted into the conventional RIE reactor to lower the dc self-bias, thus the ion energy. It is observed that the amount of bottling is less pronounced for the trenches etched with the help of a showerhead. It is believed that low energetic ions are easier reflected/captured when hitting a wall under a certain angle. So, although the angular distribution of ions coming from the plasma boundary are the same for both experiments, the energy is to small to etch the sidewall in the case of the experiments performed with showerhead.

The same effect is observed for the high density source at low pressures during changing the dc bias from 60 to 200 volts, thus indeed the ion energy is controlling bottling. When the ion energy is low enough $(<10 \mathrm{eV})$ even a very broad (i.e. high pressure) ion distribution function is completely captured by the wall or reflecting and only etching the bottom. Figure $9 \mathrm{c}$ shows a HART etched at relatively high pressure (100mTorr) with a conventional RIE.

\section{CONCLUSIONS}

A classification of the most important RIE trench effects is given. These effects can be divided into tilting and the ARDE effects i.e. bowing, bottling, ARDTOP, and RIE lag. Evidence is found that micrograss is a member of this ARDE group. It is concluded that the ion flux is the most important source of etching particles. These ions are etching the passivating layer, and are controlling the etched profile by their direction. Radicals are necessary for etching the silicon but under normal circumstances the radical density is high and they have to wait for an incoming ion before they are able to remove a silicon atom. So, to understand the RIE inhibitor process it is necessary to follow the path of an ion coming from the plasma glow.

The ion trajectory starts at the plasma boundary where the boundary distortion is influencing the electrostatic field thus the direction of the ion. During its passage through the plasma sheath, the ion will collide with other particles and its angle and energy will diverge. This effect is expressed in the ion angular/energy distribution functions (IADF and IEDF). After this the ion is entering the trench where deflection due to electrostatic fields from the conducting sample is changing the ion's direction. The ion will end its journey at the sidewall or the bottom of the trench. Depending on the energy and collision angle of the ion it will reflect, etch, or just end at the sidewall or the trench bottom. The ratio of the ions ending on the trench sidewall and bottom is directly related to RIE lag.

Tilting is caused by i) boundary distortion or ii) the local differences in radical density. Boundary distortion is found when the sample-, trench-, or wafer clamping geometry is bigger than the thickness of the sheath region. Increasing the thickness may prevent this effect and can be accomplished by lowering the pressure. A difference in the radical density between two places causes radicals to flow into the region having the smallest density. In other words, the isotropic radical flux is becoming collimated and tilting is a result.

Micrograss (fig.1c) is formed when the flux of incoming ions is highly collimated i.e. the IADF is sharp. The grass is prevented when the IADF is broadened e.g. by way of a higher pressure or due to ion reflection. In other words, a perfectly collimated ion flux is not always preferable; a little dispersion will prevent grass. 

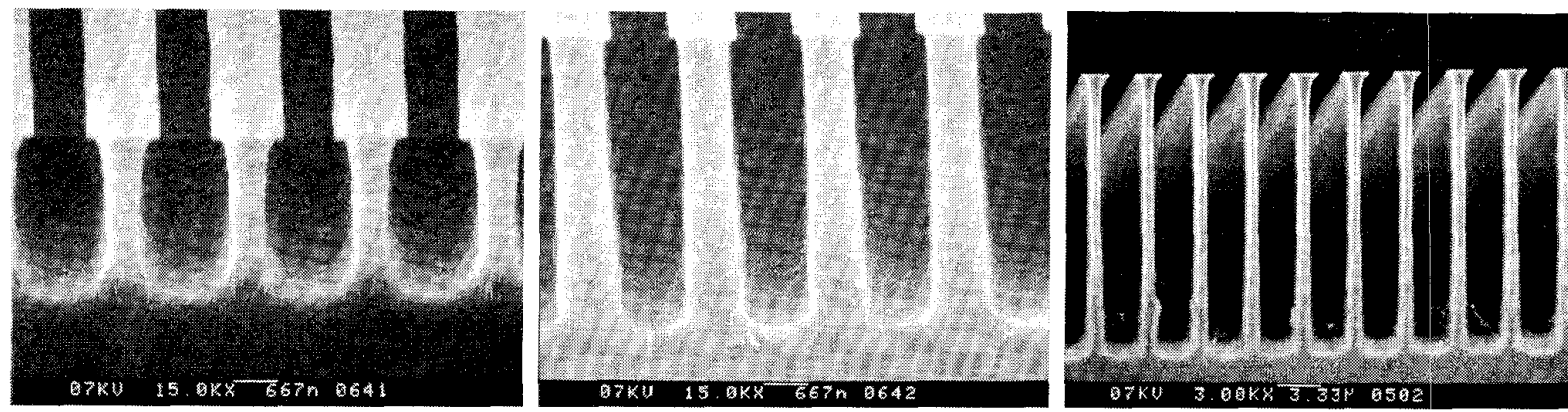

Figure 9: The influence of the ion energy on bottling: a) Without and b) with showerhead. c) HART etched with a conventional RIE.

Bowing (fig.1a) is caused by ion deflection due to electrostatic fields in the trench and the subsequent ion etching of the passivator. Increasing the passivation on the sidewall is decreasing this effect (fig.5).

TADTOP (fig. 1a) i.e. trench area dependent tapering of profiles is closely related to bowing and is found when the opposite wall of a trench is influencing the path of an ion. Therefore, when the opposite wall is close, the ion deflection is less pronounced and the tapering will be more positive. Again, increasing the sidewall passivation is decreasing this effect (fig.5).

RIE lag due to ions (fig.6) is caused by ion depletion. Ion deflection moves ions to the trench sidewall where they will be captured or etch the passivator. The amount of ions reaching the bottom will therefore be smaller. For the smaller trenches this ion depletion is reached sooner than for the wider trenches because the flux/wallarea ratio is smaller after a certain etch depth. Once again, increasing the sidewall passivation is decreasing this effect due to sidewall charging (fig.5).

RIE lag due to radicals (fig. $7 \mathrm{a} / \mathrm{b}$ ) is caused by radical depletion. The radical flux is isotropically when entering the trench but radical etching will sharpen the RADF and lower the amount of radicals what will reach the bottom. For the smaller trenches this effect is more pronounced after a certain etch depth. To decrease this effect, radical etching of the sidewall should be prevented i.e. ion deflection should be prevented. There is no prove found for RIE lag due to radical reflection.

Bottling (fig. lb) is caused by ion shadowing and sharpening of the IADF when ions are travelling down a trench. The effect is caused by off-normal ions due to a relatively high operation pressure. In the higher regions of the trench, ion are coming in at different angles and there will be a strong undercut depending on the ion energy. However, the ions which are etching the sidewall are used up and due to ion shadowing the IADF is sharpened. At a certain AR and critical angle the ions are not etching anymore but only captured or reflected. Therefore, ion shadowing and ion depletion are thought to be responsible for the bottling effect. To prevent bottling it is possible i) to sharpen the IADF by way of decreasing e.g. the pressure or ii) to decrease the IEDF by way of e.g. the dc self-bias to the point where the ions bounce with the sidewalls without etching it (fig.9). Notice that the last technique forces us to use high pressures in a conventional RIE whereas the first one indicates to use a low pressure. This paradoxical nature can be overcome by using a showerhead or a dual source system able to produce highly directional low energetic ions like the ICP-RIE.

The BSM is proven to be a practical tool to create highly anisotropic profiles with aspect ratios of at least 20 for etching HART's without sidewall bowing and with smooth bottoms and sidewalls. Especially, the low pressure cryogenically cooled ICP-RIE is a convenient apparatus to achieve this goal.

\section{ACKNOWLEDGEMENTS}

The authors thank Natlab Philips Eindhoven for supporting the silicon wafers with submicron patterns and 3 . Otter for doing the SEM work. This work is supported by the Dutch Technology Foundation (STW), which is sponsored by the Stichting voor Nederl. Wetenschappelijk Onderzoek (NWO).

\section{REFERENCES}

[1] S.M.Irving, Kodak Interface Proc., 2, 1968.

[2] R.Castaing and P.Laborie, C.R.Acad.Sci. (Paris) 238 , 1954.

[3] J.W.Coburn and H.F.Winters, J.Appl.Phys.50, 1979.

[4] A.Reinberg, U.S.Patent 3,757,733, 1973

[5] H.Jansen et al, EP appl. No. 94202519.8

[6] E.Berenschot, H.Jansen, G-J.Burger, H.Gardeniers, M.Elwenspoek, this proc.

[7] R.A.Gottscho, C.W.Jurgensen, and D.J.Vitkavage, J.Vac.Sci.Tech. B $10(5), 1992$.

[ 8] H.Jansen et al, Microelectronic Engineering 27 (1995) 475

[9] H.Jansen, H.Gardeniers, and J.Fluitman, Micromechanics Europe, Denmark, 1995.

[10]H.Jansen et al, Proc.IEEE MEMS (1995) 488

[11] J.C.Arnold and H.H.Sawin, J.Appl.Phys.70 (10), 15, 1991.

[12] S.G.Ingram, J.Appl.Phys. 68 (2), 1990.

[13] Surface Technology Systems Limited, Prince of Wales Industrial Estate, Abercarn, Newport, Gwent. NP1 5AR.UK, +44(495)249044, Fax: +44(495)249478.

[14] Oxford Instruments, Plasma Technology, North End, Yatton, Bristol BS19 4AP, England, Telephone +44(1934)876444/833851, Fax. +44(1934)834918.

[15]R.Legtenberg, H.Jansen, and M.Elwenspoek, J.Elec.Soc., 1995.

[16] W.H.Juan, S.W.Pang, A.Selvakumar, M.W.Putty, and K.Najafi, Solid-state S\$A workshop, Hilton Head, South Carolina, 1994.

[17] A.Manenschijn, Thesis, Technical University of Delft, 3 octobre 1995 